\title{
Comparison of Anthropometric Data Between Asian and Caucasian Patients With Obstructive Sleep Apnea: A Meta-Analysis
}

\author{
Jae Hoon Cho ${ }^{1, \star} \cdot$ Ji Ho Choi ${ }^{2, \star} \cdot$ Jeffrey D. Suh ${ }^{3}$ Seungho Ryu $\cdot$ Seok Hyun Cho ${ }^{5}$ \\ ${ }^{I}$ Department of Otorhinolaryngology-Head and Neck Surgery, Konkuk University College of Medicine, Seoul; ${ }^{2}$ Department of \\ Otorhinolaryngology-Head and Neck Surgery, Soonchunhyang University Bucheon Hospital, Soonchunhyang University College of Medicine, \\ Bucheon, Korea; ${ }^{3}$ Department of Head and Neck Surgery, UCLA School of Medicine, Los Angeles, CA, USA; ${ }^{4}$ Department of Occupational and \\ Environmental Medicine, Kangbuk Samsung Hospital, Sungkyunkwan University School of Medicine, Seoul; ${ }^{5}$ Department of \\ Otorhinolaryngology-Head and Neck Surgery, Hanyang University College of Medicine, Seoul, Korea
}

Objectives. Obesity is considered to be one of the most important risk factors for obstructive sleep apnea (OSA) but less is known about the role of ethnicity in OSA. The purpose of this study was to investigate the interethnic difference of obesity-related phenotypes in OSA and to reveal the role of ethnicity in OSA.

Methods. We searched MEDLINE, LILACS, Scopus, and the Cochrane Library using the key words "sleep apnea," "body mass index," "neck circumference," "waist circumference," "waist to hip ratio," etc. Inclusion criteria were adults over 18 years of age, and studies that included polysomnography, obesity-related parameters, and a clear demarcation of ethnicity in the patient population. Included studies were reviewed by 2 independent reviewers. The following information was collected for controls and OSA: number, age, gender, country, ethnicity (Asian or Caucasian), study design, apnea-hypopnea index/respiratory disturbance index, body mass index (BMI), neck circumference (NC), waist circumference (WC), and/or waist to hip ratio (WHR).

Results. A total of 8,312 publications were retrieved with a subsequent 19 manuscripts that met the selection criteria. A total of 2,966 patients were included for analysis. The main findings were as follows: There was no difference in BMI, WC, and WHR between patients with OSA and controls after accounting for publication bias; Patients with OSA have greater NC than controls (standard mean difference, $0.89 ; 95 \%$ confidence interval, 0.63 to 1.14); and There was no difference in NC between Asian and Caucasians patients $(P=0.178)$.

Conclusion. OSA might not be related with BMI, WC, and WHR. Only NC demonstrated a strong association with OSA, and this finding was not different between Asians and Caucasians.

Keywords. Sleep Apnea, Obstructive; Body Mass Index; Waist-Hip Ratio; Meta-Analysis; Obesity

\footnotetext{
- Received December 5, 2014

Revised January 19, 2015

Accepted January 26, 2015

- Corresponding author: Seok Hyun Cho

Department of Otorhinolaryngology-Head and Neck Surgery, Hanyang

University College of Medicine, 222-1Wangsimni-ro, Seongdong-gu,

Seoul 04763, Korea

Tel: +82-2-2290-8583, Fax: +82-2-2290-8588

E-mail: shcho@hanyang.ac.kr

- Co-corresponding author: Seungho Ryu

Department of Occupational and Environmental Medicine, Kangbuk

Samsung Hospital, Sungkyunkwan University School of Medicine,

29 Saemunan-ro, Jongno-gu, Seoul 03181, Korea

Tel: +82-2-2001-5137, Fax: +82-2-757-0436

E-mail: sh703.yoo@gmail.com

* The first two authors contributed equally to this study.
}

\section{INTRODUCTION}

Obstructive sleep apnea (OSA) is a common disease worldwide, affecting over $4 \%$ of men and $2 \%$ of women [1]. It is characterized by repetitive upper airway collapse during sleep, which induces frequent arousals and oxygen desaturation. OSA is associated with a variety of symptoms and conditions such as fatigue, daytime somnolence, headache, myocardial infarction, arrhythmias, stroke, and an increased incidence of motor vehicle accidents. Therefore, accurate diagnosis and treatment of OSA is recommended to improve quality of life and to reduce the risk

Copyright $\odot 2016$ by Korean Society of Otorhinolaryngology-Head and Neck Surgery

This is an open-access article distributed under the terms of the Creative Commons Attribution Non-Commercial License (http://creativecommons.org/licenses/by-nc/4.0)

which permits unrestricted non-commercial use, distribution, and reproduction in any medium, provided the original work is properly cited. 
of associated morbidity and mortality.

Numerous studies report that OSA is closely related with obesity, which is thought to reduce the diameter of upper airway and increase its collapsibility $[2,3]$. Several anthropometric measurements like body mass index (BMI), neck circumference (NC), waist circumference (WC), and waist-hip ratio (WHR) have been frequently used to investigate the association between obesity and OSA [4-22]. However, the results have varied based on age, gender, and ethnicity.

Asian patients with OSA are suspected to be affected to a greater degree by obesity than Caucasians $[23,24]$. However, this finding is not conclusive because large-scale comparisons between different ethnicities are difficult to perform at a single research center. The goal of this study was to provide a systematic and critical appraisal of the available evidence on the anthropometric differences between Asian and Caucasian patients with OSA. Understanding the different obesity phenotypes between Asian and Caucasian may help to elucidate the underlying mechanisms of OSA, provide important clinical tools for screening of OSA, and have an effect on selecting treatment modalities.

\section{MATERIALS AND METHODS}

\section{Literature sources and study identification}

This study was designed to compare the obesity-related parameters including BMI, NC, WC, and WHR between Asian and Caucasian patients with and without OSA. We searched electronic databases including MEDLINE, LILACS, Scopus, and the Cochrane Library to identify relevant articles published in English from 1970 to October 2013. The following key words were used: sleep apnea AND body mass index; sleep apnea AND neck circumference; sleep apnea AND waist circumference; sleep apnea AND waist to hip ratio; sleep apnea AND central obesity; sleep apnea AND anthropometry. Only manuscripts published in English were eligible. Reviews, guidelines, letters, case reports, editorials, in vitro and animal studies were excluded. Data was extracted from the final group of included studies using a standardized datasheet. This was performed independently in duplicate (by authors JHC and SHC) and all authors reconciled the results.

\section{Eligibility criteria}

All case-control, cross-sectional, and cohort studies were included if the study population was limited to adults older than 18 years, a laboratory polysomnography or home sleep test was performed, obesity-related parameters (BMI, NC, WC, and/or WHR) were measured, and there was a clear demarcation of ethnicity, especially in countries with mixed ethnic populations such as in United States. We excluded studies which did not provide raw data on apnea-hypopnea index (AHI)/respiratory disturbance index (RDI) and obesity-related parameters (BMI, NC, WC, and/or WHR) with mean and standard deviations. We also excluded studies which included cases of pregnancy, craniofacial deformity, and bariatric surgery.

\section{Data collection}

For each study, the following information was collected: number of patients and controls, age, gender, country, ethnicity (Asian or Caucasian), study design, AHI/RDI, BMI, NC, WC, and/or WHR. According to the map of ethnic distribution based on genetic similarity, we classified materials into 2 ethnic groups; 9 Asians (Korea, China, Japan, and Thailand) and 10 Caucasians (United States, Italy, Spain, Turkey, and India) [25]. If two or more studies presented the same data from a single patient population, we included the data in our analysis only once.

\section{Statistical analyses}

BMI, NC, WC, and WHR were statistically analyzed totally and separately based on ethnicity, specifically between Asian and Caucasian groups. We used the random-effects model according to Dersimonian-Laird and the fixed effects model according to the inverse variance approach simultaneously irrespective of heterogeneity, and provided both results. Heterogeneity was calculated with the Cochran's Q statistic test and the $\mathrm{I}^{2}$ test. We considered an $\mathrm{I}^{2}$ value greater than $50 \%$ and $P<0.05$ as indicative of heterogeneity. Subgroup data was compared to explore the differences between Asian and Caucasian. If the heterogeneity existed between groups, we considered that there was difference between the two. Publication bias was examined by visual inspection of a funnel plot and Egger's test for bias [26]. Publication bias is suspected when the funnel plot is asymmetrical. We also performed the Duval and Tweedie "trim-and-fill" procedure to further assess the possible effect of publication bias in our meta-analysis [27]. Analysis was performed using STATA ver. 13 (StataCorp LP., College Station,TX, USA). All results are reported with standard mean difference (SMD) and 95\% confidence intervals (CIs). All $P$-values were two-tailed.

\section{Search results and characteristics}

A total of 8,312 manuscripts were retrieved. A systematic review of titles, abstracts, and full text publications was carried out. 8,260 studies were discarded and 52 studies were selected for the further review. Thirty-three studies were abandoned due to lack of raw data or discordance of inclusion criteria. Finally, 19 studies remained for data extraction. The search results are summarized in Fig. 1.

\section{RESULTS}

This meta-analysis included 19 studies consisting of data from a total of 2,966 patients (803 controls and 2,163 with OSA,Table 1). Males represented $57.9 \%$ of controls (range, $8.3 \%$ to $90 \%$ ), and $76.5 \%$ of patients with OSA (range, $40.4 \%$ to $90 \%$ ). For 
controls and OSA group, there was no statistical difference in sex and age between Asians and Caucasians. Sixteen studies $(84.2 \%)$ defined the presence of OSA with the criteria of AHI $\geq$ 5 , and 17 studies $(89.5 \%)$ performed standard in-laboratory full-night polysomnography.

\section{BMI between Asians and Caucasians}

There was a significant heterogeneity among all the studies $\left(\mathrm{I}^{2}=74.2 \%, P<0.001\right)$, so we used a random effects model to derive the overall pooled estimates which showed that patients with OSA had greater BMI than controls (SMD, 0.62; 95\% CI, 0.44

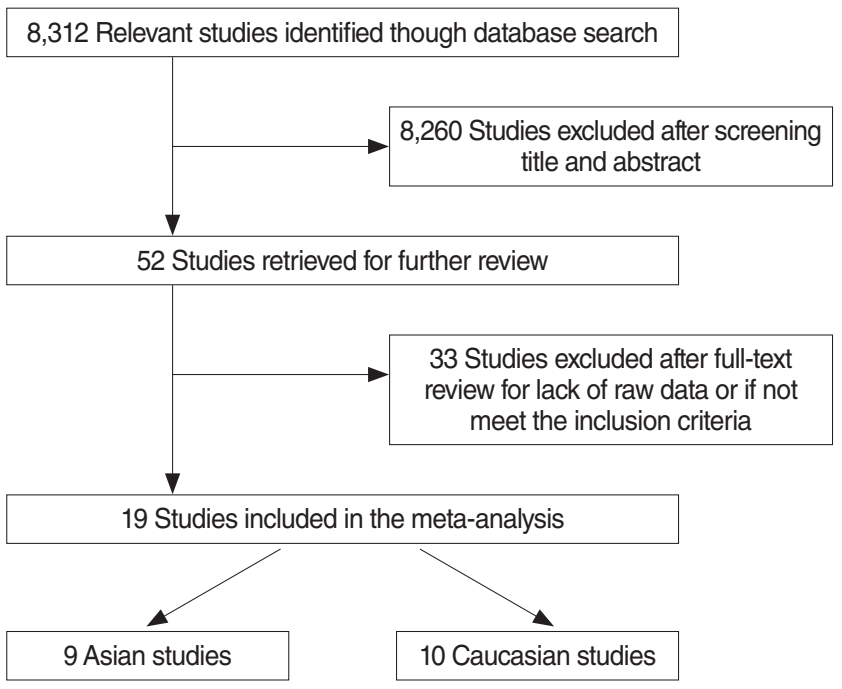

Fig. 1. Search results. to 0.80; Fig. 2). However, funnel plots for BMI was asymmetric (Fig. 3) and Egger test suggested publication bias $(P=0.007)$. Therefore, trim and fill analysis based on a random effects model was done to revise the publication bias. These results showed that BMI was no longer different between OSA patients and controls (SMD, $1.2 ; 95 \%$ CI, -0.40 to 2.79 ).

\section{$\mathrm{NC}$ between Asians and Caucasians}

There was significant heterogeneity among the studies $\left(\mathrm{I}^{2}=81.0 \%\right.$, $P<0.001$ ), so we used a random effects model to derive the overall pooled estimates. This showed that patients with OSA had greater NC than control (SMD, 0.89; 95\% CI, 0.63 to 1.14; Fig. 2). The funnel plot for NC was symmetrical (Fig. 3) and the Egger test for publication bias was also not significant $(P=0.769)$. For subgroup analysis, we also used fixed effect model for Asians $\left(I^{2}=44.9 \%\right)$ and random effect model for Caucasians $\left(I^{2}=90.0 \%\right)$. Subgroup analysis also showed that patients with OSA had similar NC compared to controls for both Asians (SMD, 0.75; 95\% CI, 0.53 to 0.98 ) and Caucasians (SMD, 0.939; 95\% CI, 0.79 to 1.07). Heterogeneity between 2 subgroups was not significant $(P=0.178)$, which means that the association between $\mathrm{NC}$ and OSA did not differ by the ethnicity.

\section{WC between Asians and Caucasians}

There was also significant heterogeneity among the studies $\left(\mathrm{I}^{2}=67.4 \%, P<0.001\right)$. We used a random effects model to derive the overall pooled estimates. This showed that patients with OSA had greater WC than controls (SMD, 0.81; 95\% CI, 0.64 to 0.99 ; Fig. 4). However, funnel plot for WC was asymmetric

Table 1. Characteristics of included study to evaluate the ethnicity and obesity in controls and patients with obstructive sleep apnea

\begin{tabular}{|c|c|c|c|c|c|c|c|}
\hline Source & Country & No. of subjects & $\mathrm{AHI}$ & $\mathrm{BMl}\left(\mathrm{kg} / \mathrm{m}^{2}\right)$ & $\mathrm{NC}$ & WC & WHR \\
\hline Ip et al. [4] & China & $30 / 30$ & $1.8 / 35.7$ & $26.5 / 27$ & $38.5 / 38.9$ & $89.9 / 91.9$ & $0.9 / 0.91$ \\
\hline Yucel et al. [5] & Turkey & $24 / 47$ & $2.1 / 29.6$ & $34.1 / 32.9$ & $37.4 / 39.3$ & NR & NR \\
\hline Julia-Serda et al. [6] & Spain & $92 / 115$ & $3 / 46$ & $29.7 / 32.6$ & $40.6 / 43.5$ & $102.5 / 111.4$ & NR \\
\hline Zhao et al. [7] & China & $36 / 32$ & $5 / 32$ & $23 / 26$ & $38.1 / 40.3$ & NR & $0.93 / 0.98$ \\
\hline Sharma et al. [8] & India & $80 / 40$ & $1 / 32.2$ & 25.3/29.8 & $36.3 / 39.3$ & $92.7 / 106.9$ & $0.85 / 1$ \\
\hline Liu et al. [9] & China & $18 / 58$ & $5.7 / 37$ & 25.6/28.4 & $37.6 / 40.1$ & NR & NR \\
\hline Martinez-Rivera et al. [10] & Spain & $68 / 124$ & $4.9 / 38.9$ & $31 / 31.4$ & $40.5 / 42.5$ & $100.7 / 106.3$ & $0.94 / 0.98$ \\
\hline Nakagawa et al. [11] & Japan & $18 / 75$ & $2.4 / 36$ & 23.8/29.2 & NR & $86.7 / 97.7$ & NR \\
\hline Jelic et al. [12] & USA & $33 / 38$ & $1 / 36$ & $30 / 35$ & NR & $96.5 / 109.2$ & $0.88 / 0.93$ \\
\hline Angelico et al. [13] & Italy & $48 / 178$ & $1.5 / 31.4$ & 29.2/32.1 & NR & $101.2 / 110.1$ & $0.94 / 0.97$ \\
\hline Ugur et al. [14] & Turkey & $24 / 73$ & $2.6 / 18.7$ & $29 / 32.4$ & $42 / 42$ & $99 / 101$ & NR \\
\hline Kawaguchi et al. [15] & Japan & $30 / 189$ & $2.3 / 28.9$ & 23.1/27.3 & $36.8 / 40.3$ & $79.3 / 91.8$ & NR \\
\hline Soylu et al. [16] & Turkey & $68 / 431$ & $2.3 / 36.5$ & $26.9 / 32.2$ & $37.7 / 43$ & $96 / 113.1$ & NR \\
\hline Lubrano et al. [17] & Italy & $67 / 104$ & $1.5 / 27.2$ & $35.7 / 43.4$ & NR & $111.5 / 129.2$ & $0.96 / 0.99$ \\
\hline Lin et al. [18] & China & $45 / 113$ & $3.3 / 23$ & $22.4 / 22.7$ & NR & $79.9 / 81.4$ & $0.94 / 0.94$ \\
\hline Apaydin et al. [19] & Turkey & $20 / 67$ & $2.4 / 47.6$ & $27.4 / 31.6$ & $30 / 41$ & $98.5 / 109.7$ & $0.94 / 0.99$ \\
\hline Liu et al. [20] & China & $19 / 130$ & $3.2 / 30.9$ & $24.4 / 28$ & $36.2 / 39.9$ & NR & NR \\
\hline Banhiran et al. [21] & Thailand & $66 / 217$ & $1.9 / 34.3$ & $25.3 / 28.4$ & $35.1 / 38.5$ & $86.4 / 96$ & NR \\
\hline Lim et al. [22] & Korea & $17 / 102$ & $1.9 / 36.9$ & $25.6 / 26.6$ & $37.7 / 40.3$ & $89.9 / 96.1$ & $0.9 / 0.95$ \\
\hline
\end{tabular}

Values are presented as control/obstructive sleep apnea.

$\mathrm{AHI}$, apnea-hypopnea index; BMI, body mass index; NC, neck circumference; WC, waist circumference; WHR, waist-hip ratio; NR, not recorded. 


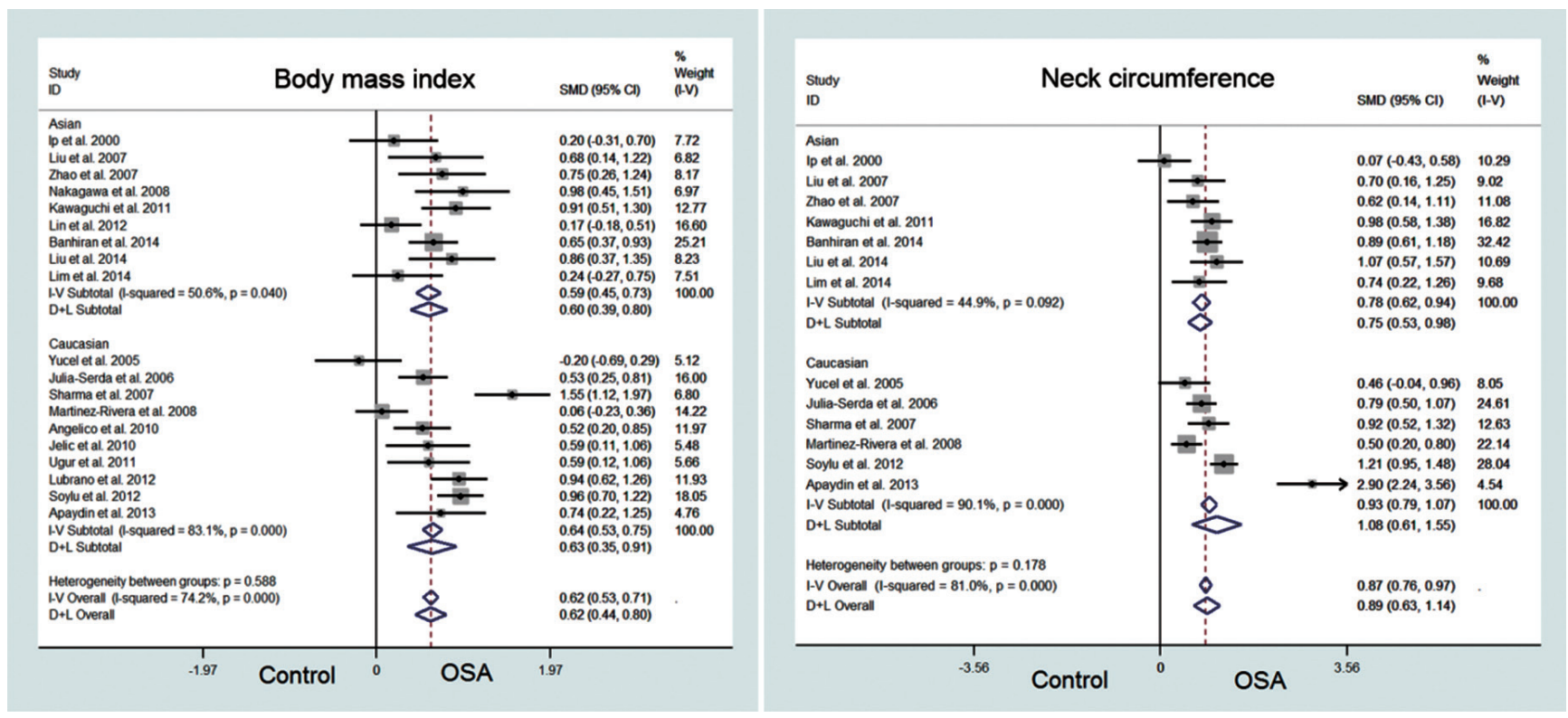

Fig. 2. Forest plot for body mass index and neck circumference. OSA, obstructive sleep apnea; SMD, standard mean difference; Cl, confidence inerval.

Funnel plot with pseudo $95 \%$ confidence limits
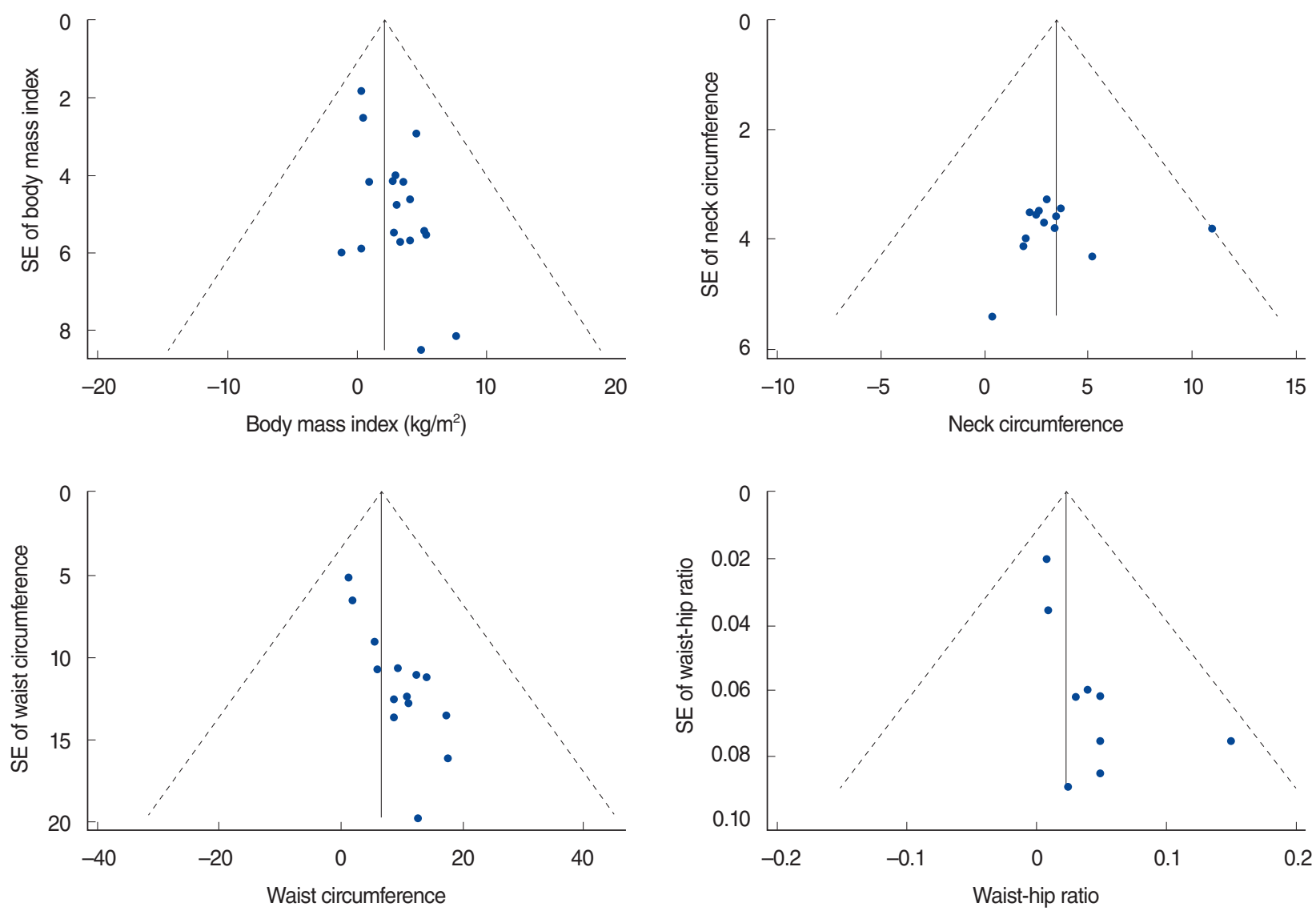

Fig. 3. Funnel plot for body mass index, neck circumference, waist circumference, and waist-hip ratio. SE, standard error. 

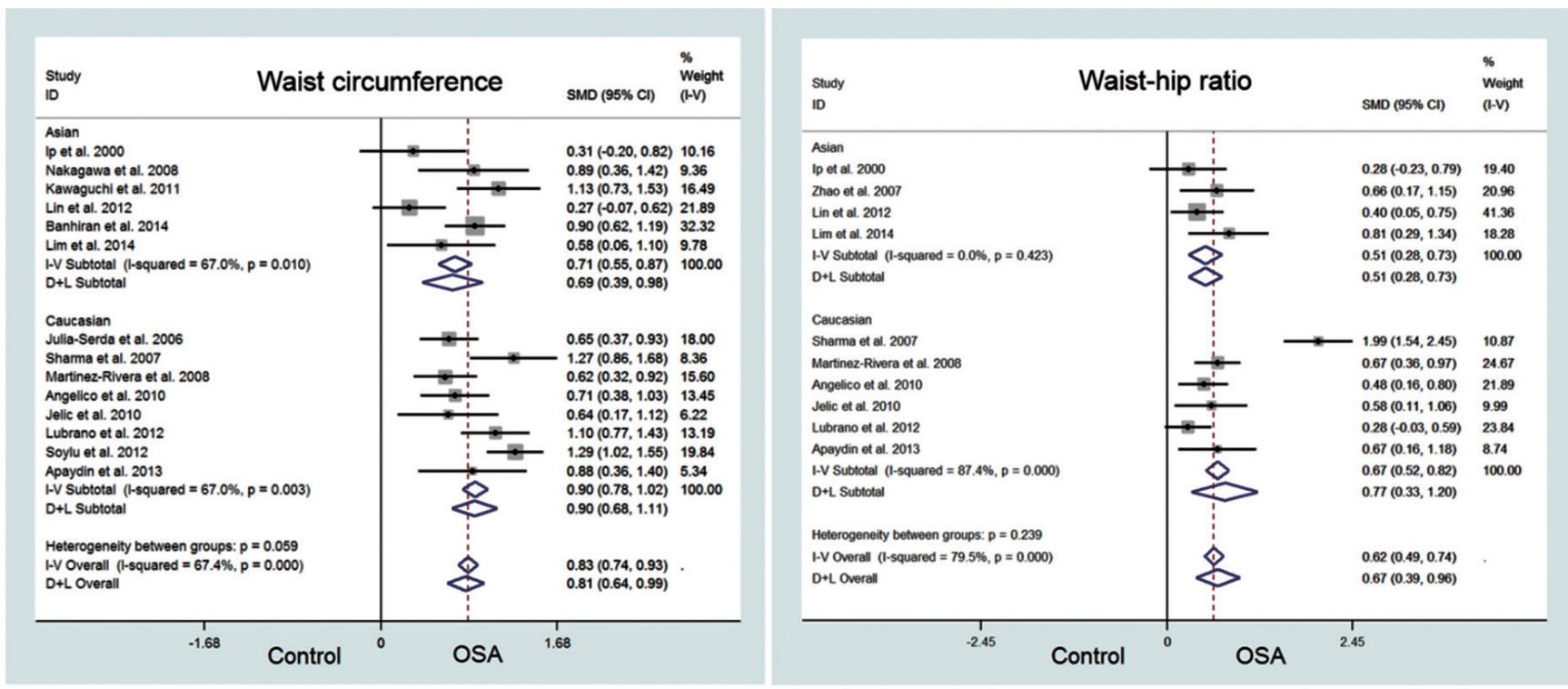

Fig. 4. Forest plot for waist circumference and waist-hip ratio. OSA, obstructive sleep apnea; SMD, standard mean difference; Cl, confidence inerval.

(Fig. 3) and Egger test suggested publication bias $(P<0.001)$. Therefore, trim and fill analysis based on a random effects model was done to revise the publication bias and the results showed that WC was not different between OSA patients and controls (SMD, 3.97; $95 \%$ CI, -0.58 to 8.53 ).

\section{WHR between Asians and Caucasians}

There was significant heterogeneity among the studies $\left(\mathrm{I}^{2}=79.5 \%\right.$, $P<0.001)$ when WHR was examined. A random effects model was used to derive the overall pooled estimates which showed that patients with OSA had greater WHR than controls (SMD, 0.67; 95\% CI, 0.39 to 0.96; Fig. 4). However, the funnel plot for WHR as asymmetric (Fig. 3) and Egger test suggested publication bias $(P=0.018)$. Therefore, trim and fill analysis based on a random effects model was done to revise the publication bias. The result showed that WHR was no longer significant between OSA patients and controls (SMD, $0.01 ; 95 \% \mathrm{CI},-0.01$ to 0.04 ).

\section{DISCUSSION}

There are various pathophysiologic factors associated with OSA such as obesity, unfavorable anatomical features, neuromuscular abnormalities, or genetic problems. Of these, obesity is thought to be one of the most common and important risk factors for OSA. Obesity is associated with increased narrowing and obstruction of the airway due to changes at the level of the soft palate, pharyngeal wall, and tongue base [28]. In addition, obesity may lead to modifications of neuromuscular function resulting in increased airway collapsibility and weakness of neuromuscular compensation $[3,29]$.
The objective of this study was to identify any potential ethnicity-specific characteristics of obesity-related anthropometric measurements (BMI, NC,WC, andWHR). We performed a schematic meta-analysis by stratifying the raw data by ethnicity (Asian vs. Caucasian). The key findings of this study were that: (1) BMI, WC, and WHR were not different between patients with OSA and controls after evaluating for publication bias; (2) Patients with OSA had greater NC than controls in both Asians and Caucasians; and (3) Anthropometric phenotypes (BMI, NC, WC, and WHR) of Asian OSA patients were not different from Caucasian OSA patients. Our study results provide valuable data for clinicians when evaluating patients with different ethnicities with suspected OSA by identifying anthropometric characteristics associated with OSA that can be used to assist with the diagnosis prior to a standard polysomnography.

Previous studies have reported that obesity-related measurements such as BMI,WC, and WHR are significant risk factors for OSA [2,3]. Surprisingly, our meta-analysis found no difference in BMI, WC, and WHR between patients with OSA and controls when publication bias was considered. Only NC was greater and statistically significant among OSA patients regardless of the ethnicity. We used the 'trim and fill method' described by Duval and Tweedie [27] to identify and reconcile potential publication bias. This removes (trims) the smaller studies causing funnel plot asymmetry, and uses the trimmed funnel plot to estimate the true center of the funnel. Omitted studies and their missing counterparts are replaced around the center (fill). The results of this analysis are somewhat surprising, but raises the possibility that some of the anthropometric features of obesity, namely BMI,WC, and WHR, should be evaluated more carefully as true risk factors for OSA in future studies. Our findings may indicate that many non- 
obese patients with OSA are still unrecognized or undiagnosed.

Among the four anthropometric measurements evaluated in our analysis, only NC was found to be statistically significant between OSA patients and controls. Although obesity is often regarded as a principle risk factor in the occurrence of OSA, our results suggest that the $\mathrm{NC}$ is more closely correlated with OSA without publication bias. Interestingly, we found no difference in NC between Asian and Caucasian patients with OSA and controls. Several studies report that Asians have more severe OSA than Caucasians. Ong et al. [30] evaluated demographic and polysomnographic data between Caucasians and Asians, and found that Asians with OSA had more severe disease than Caucasians when matched for age, gender, and BMI. And Li et al. [31] found that despite having a lower BMI than Caucasians, Asian men had more severe OSA. In this study, we investigated whether differences in obesity phenotypes between Asian and Caucasians were risk factors in OSA. However, we found that most of anthropometric data (BMI, WC, and WHR) lack of confidence due to publication bias and did not show interethnic differences. NC was the most reliable measurement and most likely represents a true risk factor for OSA in both Asian and Caucasian patients

There are several limitations of this study. The trim and fill method was used to assess if publication bias was present and to estimate the effect when the bias is removed. It is one of the most common statistical methods used to resolve potential publication bias in meta-analysis, and the method has been found to be accurate [32]. However, it is not the only method available, and it requires several statistical assumptions, including that funnel plot asymmetry is caused solely by publication bias. Further studies are required to confirm our results. Finally, sex, age, and severity of OSA were not included in this meta-analysis due to lack of data in the reviewed studies.

In conclusion, obstructive sleep apnea is an airway disease known to be closely related to obesity. However, our study found that the many of the features of obesity such as BMT,WC, and WHR might not be significant risk factors for OSA due to publication bias. In this study, NC demonstrated a strong association with OSA, and this finding was not different between Asians and Caucasians.

\section{CONFLICT OF INTEREST}

No potential conflict of interest relevant to this article was reported.

\section{ACKNOWLEDGMENTS}

This article was supported by research funding from Hanyang University (HY-2013).

\section{REFERENCES}

1. Young T, Palta M, Dempsey J, Skatrud J, Weber S, Badr S. The occurrence of sleep-disordered breathing among middle-aged adults. N Engl J Med. 1993 Apr;328(17):1230-5.

2. Vgontzas AN. Does obesity play a major role in the pathogenesis of sleep apnoea and its associated manifestations via inflammation, visceral adiposity, and insulin resistance? Arch Physiol Biochem. 2008 Oct;114(4):211-23.

3. Namyslowski G, ScierskiW, Mrowka-Kata K, Kawecka I, Kawecki D, Czecior E. Sleep study in patients with overweight and obesity. J Physiol Pharmacol. 2005 Dec;56 Suppl 6:59-65.

4. Ip MS, Lam KS, Ho C, Tsang KW, Lam W. Serum leptin and vascular risk factors in obstructive sleep apnea. Chest. 2000 Sep;118(3):580-6.

5. Yucel A, Unlu M, Haktanir A, Acar M, Fidan F. Evaluation of the upper airway cross-sectional area changes in different degrees of severity of obstructive sleep apnea syndrome: cephalometric and dynamic CT study.AJNRAm J Neuroradiol. 2005 Nov-Dec;26(10):2624-9.

6. Julia-Serda G, Perez-Penate G, Saavedra-Santana P, Ponce-Gonzalez M, Valencia-Gallardo JM, Rodriguez-Delgado R, et al. Usefulness of cephalometry in sparing polysomnography of patients with suspected obstructive sleep apnea. Sleep Breath. 2006 Dec;10(4):181-7.

7. Zhao ZH, Sullivan C, Liu ZH, Luo Q, Xiong CM, Ni XH, et al. Prevalence and clinical characteristics of sleep apnea in Chinese patients with heart failure. Int J Cardiol. 2007 May;118(1):122-3.

8. Sharma SK, Kumpawat S, Goel A, Banga A, Ramakrishnan L, Chaturvedi P. Obesity, and not obstructive sleep apnea, is responsible for metabolic abnormalities in a cohort with sleep-disordered breathing. Sleep Med. 2007 Jan;8(1):12-7.

9. Liu KH, Chu WC, To KW, Ko FW, Tong MW, Chan JW, et al. Sonographic measurement of lateral parapharyngeal wall thickness in patients with obstructive sleep apnea. Sleep. 2007 Nov;30(11):1503-8.

10. Martinez-Rivera C, Abad J, Fiz JA, Rios J, Morera J. Usefulness of truncal obesity indices as predictive factors for obstructive sleep apnea syndrome. Obesity (Silver Spring). 2008 Jan;16(1):113-8.

11. Nakagawa Y, Kishida K, Kihara S, Sonoda M, Hirata A, Yasui A, et al. Nocturnal reduction in circulating adiponectin concentrations related to hypoxic stress in severe obstructive sleep apnea-hypopnea syndrome. Am J Physiol Endocrinol Metab. 2008 Apr;294(4):E778-84.

12. Jelic S, Lederer DJ, Adams T, Padeletti M, Colombo PC, Factor PH, et al.Vascular inflammation in obesity and sleep apnea. Circulation. 2010 Mar;121(8):1014-21.

13. Angelico F, del Ben M, Augelletti T, de Vita R, Roma R, Violi F, et al. Obstructive sleep apnoea syndrome and the metabolic syndrome in an internal medicine setting. Eur J Intern Med. 2010 Jun;21(3): 191-5.

14. Ugur KS, Ark N, Kurtaran H, Kizilbulut G, Cakir B, Ozol D, et al. Subcutaneous fat tissue thickness of the anterior neck and umbilicus in patients with obstructive sleep apnea. Otolaryngol Head Neck Surg. 2011 Sep;145(3):505-10.

15. Kawaguchi Y, Fukumoto S, Inaba M, Koyama H, Shoji T, Shoji S, et al. Different impacts of neck circumference and visceral obesity on the severity of obstructive sleep apnea syndrome. Obesity (Silver Spring). 2011 Feb;19(2):276-82.

16. Soylu AC, Levent E, Sariman N, Yurtlu S, Alparslan S, Saygı A. Obstructive sleep apnea syndrome and anthropometric obesity indexes. Sleep Breath. 2012 Dec;16(4):1151-8.

17. Lubrano C, Saponara M, Barbaro G, Specchia P, Addessi E, Costantini D, et al. Relationships between body fat distribution, epicardial fat and obstructive sleep apnea in obese patients with and without metabolic syndrome. PLoS One. 2012;7(10):e47059.

18. Lin QC, Zhang XB, Chen GP, Huang DY, Din HB, Tang AZ. Obstructive sleep apnea syndrome is associated with some components of 
metabolic syndrome in nonobese adults. Sleep Breath. 2012 Jun; 16(2):571-8.

19. Apaydin M,Ayik SO,Akhan G, Peker S, Uluc E. Carotid intima-media thickness increase in patients with habitual simple snoring and obstructive sleep apnea syndrome is associated with metabolic syndrome. J Clin Ultrasound. 2013 Jun;41(5):290-6

20. Liu KH, ChuWC, To KW, Ko FW, Ng SS, Ngai JC, et al. Mesenteric fat thickness is associated with increased risk of obstructive sleep apnoea. Respirology. 2014 Jan;19(1):92-7.

21. Banhiran W, Junlapan A, Assanasen P, Chongkolwatana C. Physical predictors for moderate to severe obstructive sleep apnea in snoring patients. Sleep Breath. 2014 Mar;18(1):151-8.

22. Lim YH, Choi J, Kim KR, Shin J, Hwang KG, Ryu S, et al. Sex-specific characteristics of anthropometry in patients with obstructive sleep apnea: neck circumference and waist-hip ratio. Ann Otol Rhinol Laryngol. 2014 Jul;123(7):517-23.

23. Lam B, Lam DC, Ip MS. Obstructive sleep apnoea in Asia. Int J Tuberc Lung Dis. 2007 Jan;11(1):2-11.

24. Villaneuva AT, Buchanan PR, Yee BJ, Grunstein RR. Ethnicity and obstructive sleep apnoea. Sleep Med Rev. 2005 Dec;9(6):419-36.

25. Cavalli-Sforza LL, Menozzi P, Piazza A. The history and geography of human genes. Princeton (NJ): Princeton University Press; 1994.
26. Egger M, Davey Smith G, Schneider M, Minder C. Bias in meta-analysis detected by a simple, graphical test. BMJ. 1997 Sep;315(7109): 629-34.

27. Duval S, Tweedie R. Trim and fill: a simple funnel-plot-based method of testing and adjusting for publication bias in meta-analysis. Biometrics. 2000 Jun;56(2):455-63.

28. Young T, Peppard PE, Taheri S. Excess weight and sleep-disordered breathing. JAppl Physiol (1985). 2005 Oct;99(4):1592-9.

29. Fogel RB, MalhotraA, White DP. Sleep. 2: pathophysiology of obstructive sleep apnoea/hypopnoea syndrome. Thorax. 2004 Feb;59(2): 159-63.

30. Ong KC, Clerk AA. Comparison of the severity of sleep-disordered breathing in Asian and Caucasian patients seen at a sleep disorders center. Respir Med. 1998 Jun;92(6):843-8.

31. Li KK, Kushida C, Powell NB, Riley RW, Guilleminault C. Obstructive sleep apnea syndrome: a comparison between Far-East Asian and white men. Laryngoscope. 2000 Oct;110(10 Pt 1):1689-93.

32. Peters JL, Sutton AJ, Jones DR, Abrams KR, Rushton L. Performance of the trim and fill method in the presence of publication bias and between-study heterogeneity. Stat Med. 2007 Nov;26(25): 4544-62. 